CASE REPORT

\title{
Fatal fulminant myocarditis caused by disseminated mucormycosis
}

\author{
A Basti, S Taylor, M Tschopp, J Sztajzel
}

Heart 2004;90:e60 (http://www.heartjnl.com/cgi/content/full/90/10/e60). doi: 10.1136/hrt.2004.038273

Acute fulminant myocarditis is a critical clinical condition with sudden onset of severe congestive heart failure followed by severe haemodynamic deterioration. Instituting early left ventricular support may improve outcome and result in better long term survival. The case of an immunocompromised patient who developed acute fulminant myocarditis in the setting of disseminated mucormycosis is presented.

\section{CASE REPORT}

A 40 year old woman with aplastic anaemia treated with immunosuppressive agents and steroids, who had undergone a medullary cell transplant in February 2003, was admitted to our hospital on 12 April 2003 because of fatigue, nausea, vomiting, dehydration, and renal insufficiency. The patient subsequently developed a staphylococcal bacteraemia, which was treated with antibiotics; however, she continued to have fever on the following days. Therefore, an echocardiogram was recorded, which was normal. On 27 April, ST segment elevation was observed on continuous ECG monitoring, although the patient was asymptomatic. A 12 lead ECG (fig 1) showed concave ST elevation in almost all leads. The second echocardiogram showed a normal left ventricular cavity without hypertrophy and preserved systolic function, no valvar vegetations, no pulmonary hypertension, and no significant pericardial effusion. Because of the increase of cardiac enzymes (creatine kinase $\mathrm{MB}$ and troponin) the patient was taken to the intensive care unit with a diagnosis of myopericarditis.
During the night the patient rapidly developed severe heart failure. The echocardiogram recorded at that time, eight hours after the second one, showed severe dilatation of the left ventricle with akinesia of almost all segments and severe systolic dysfunction. The patient had cardiopulmonary arrest and electromechanical dissociation, necessitating cardiopulmonary resuscitation. Because of the patient's general poor condition, including pancytopenia, septicaemia, and severe hypoxaemia, we decided against providing mechanical circulatory support. The patient died after 90 minutes of cardiopulmonary resuscitation.

Necropsy showed disseminated mucormycosis in the lungs, heart, liver, kidneys, brain, and skin. Macroscopically and histologically, there was mycotic infiltration of the pericardium (fig 2A, B) and of the myocardium with zones of freshly infarcted tissue (fig 3A, B).

\section{DISCUSSION}

This immunocompromised patient died of an acute fulminant myocarditis in the setting of disseminated mucormycosis. Mucormycosis is a fungal infection and may affect immunocompromised patients with haematological malignancies. ${ }^{12}$ Because of its variable clinical manifestations mucormycosis is not diagnosed during life, rendering the disease most often fatal, although in a few cases successful treatment has been reported. ${ }^{3}$

In our patient, the fungal infection had disseminated into the pericardium and the myocardium with rapid deterioration of left ventricular function. Clinically, we initially observed only the presence of ST segment elevation on the ECG, with no particular symptoms. Possible diagnoses for these ECG changes were acute myocardial infarction and acute pericarditis associated or not with myocarditis (fig 4).

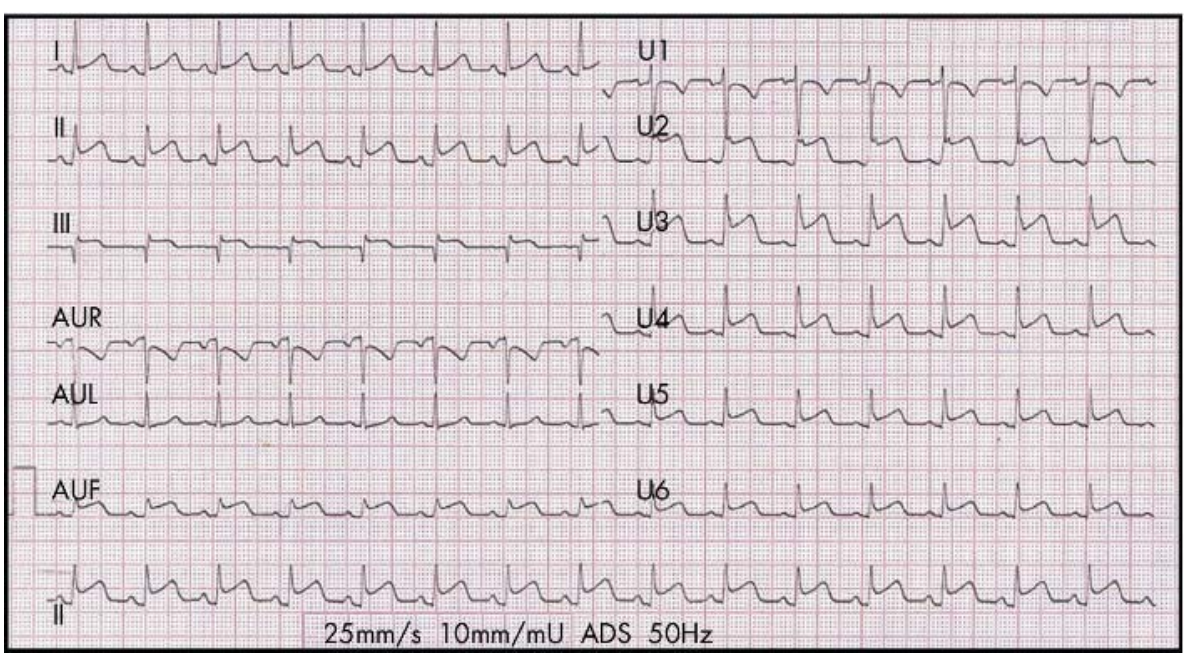

Figure 1 Twelve lead ECG showing concave ST elevation in almost all leads. 


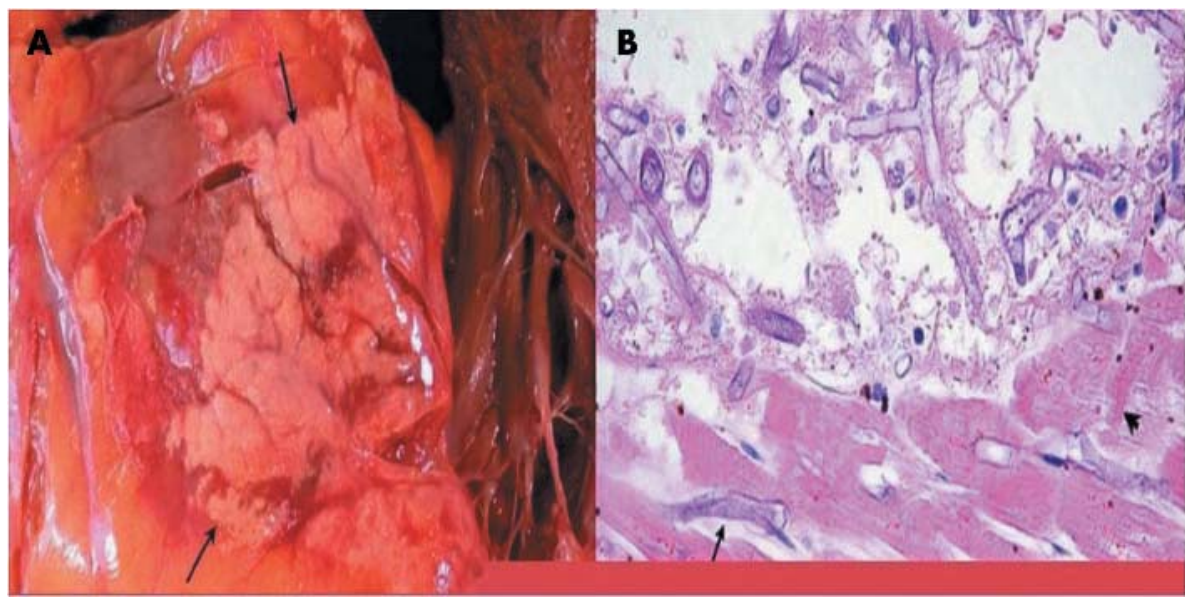

Figure 2 (A) Epicardial necrotic plaques (arrows) caused by mycotic embolisation. (B) Mycelium (arrow) crossing the vascular wall and infiltrating the myocardium. Contraction bands correspond to necrotic myocytes.

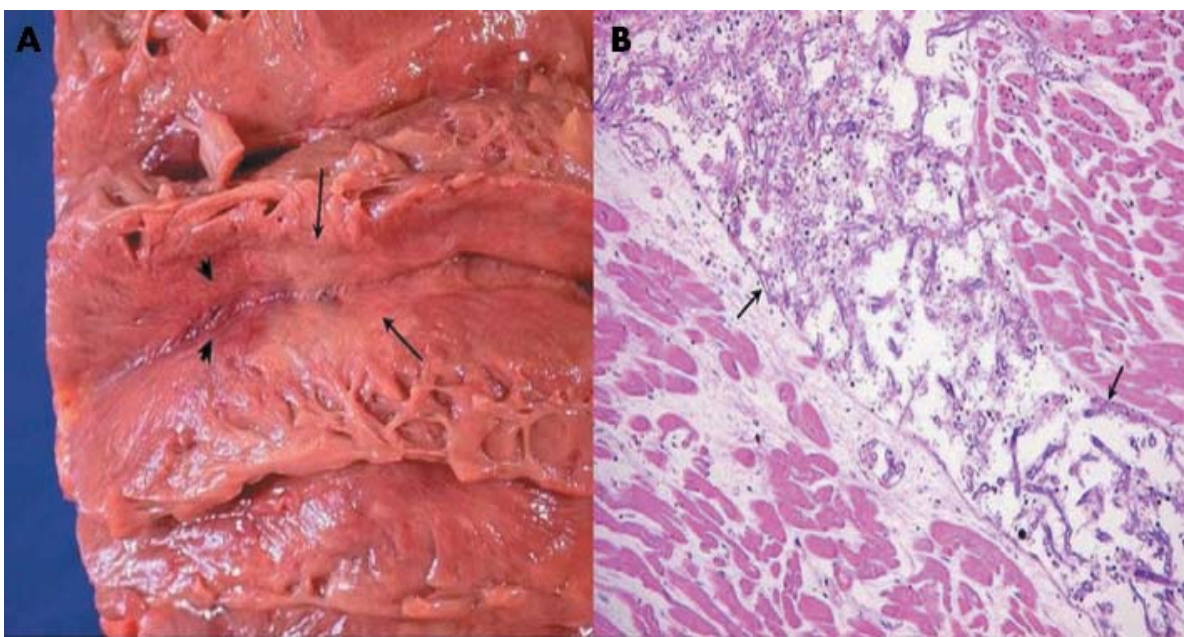

Figure 3 (A) White zones (arrows) of mycotic infiltration and red zones (arrowheads) of freshly infarcted zones. (B) Irregular tubular structures corresponding to mycotic filaments (arrows) in intramyocardial vessels.
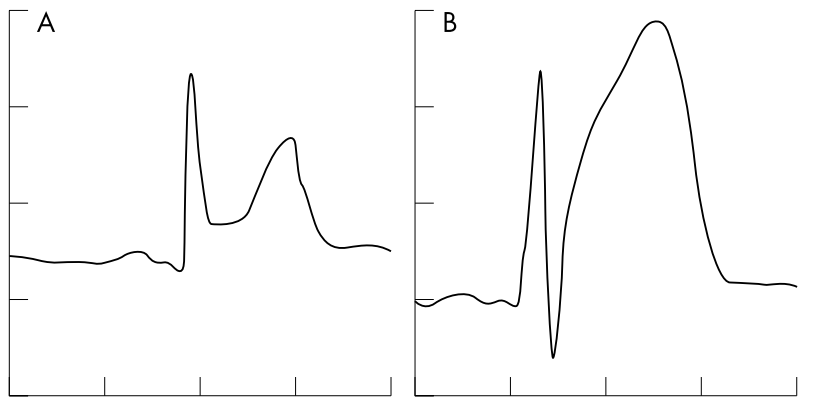

Figure 4 (A) Concave ST elevation caused by acute pericarditis. (B) Convex ST elevation in the setting of acute transmural ischaemia.

Our patient had concave ST elevation with depression of the PR interval, most probably corresponding to acute pericarditis. Because cardiac enzyme concentrations were increased myopericarditis was diagnosed. A coronary angiogram was not made because the patient had no chest pain and because of the very low probability of coronary artery disease.

Acute myocarditis is an inflammatory disease of the myocardium and may be of a common or a fulminant type. Acute fulminant myocarditis is characterised by notable myocardial inflammation with sudden onset of severe congestive heart failure followed by severe haemodynamic deterioration. ${ }^{4}$ Although acute fulminant myocarditis is a critical clinical condition at presentation, data from a study ${ }^{5}$ have shown that patients with this disorder may have a better long term survival than those with acute common myocarditis. Echocardiographic findings in patients with fulminant myocarditis at presentation have shown a nondilated left ventricular cavity with a thickened and hypocontractile left ventricle. Patients with acute common myocarditis had a dilated left ventricle, with normal thickness, but decreased systolic function. ${ }^{6}$ These findings may be one of the reasons for the better prognosis during follow up of patients with acute fulminant myocarditis. Taking into account these data some authors have suggested the use of early short term circulatory support with left ventricular assist devices combined with intensive medical treatment. ${ }^{78}$

The case of our patient illustrates the very rapid and dramatic deterioration of left ventricular function, which had still been normal several hours earlier, and raises the question of what is the threshold for instituting early ventricular support, particularly in very ill patients.

\section{Authors' affiliations}

A Basti, J Sztajzel, Cardiology Center, University Hospital Geneva, Geneva, Switzerland

S Taylor, Department of Pathology, University Hospital Geneva

M Tschopp, Medical Clinics, Department of Internal Medicine, University Hospital Geneva 
Correspondence to: Dr Juan Sztajzel, Cardiology Center, University Hospital Geneva, 24, rue Micheli-du-Crest, 1211 Geneva 4, Switzerland; juan.sztajzel@hcuge.ch

Accepted 28 April 2004

\section{REFERENCES}

1 Sugar AM. Agents of mucormycosis and related species. In: Mandell Gl Bennett JE, Dolin R, eds. Principles and practice of infectious diseases. New York: Churchill Livingstone, 1995:2311-21.

2 Cuvelier I, Vogelaers D, Peleman R, et al. Two cases of disseminated mucormycosis in patients with hematological malignancies and literature review. Eur J Clin Microbiol Infect Dis 1998;17:859-63.
3 Gonzalez CE, Couriel DR, Walsh TJ. Disseminated zygomycosis in a neutropenic patient: successful treatment with amphotericin B lipid complex and granulocyte colony-stimulating factor. Clin Infect Dis 1997;24:192-6.

4 Feldman AM, McNamara D. Myocarditis. N Engl J Med 2000;343:1388-98.

5 McCarthy RE III, Boehmer JP, Hruban RH, et al. Long-term outcome of fulminant myocarditis as compared with acute (nonfulminant) myocarditis. N Engl J Med 2000;342:690-5.

6 Felker GM, Boehmer JP, Hruban RH, et al. Echocardiographic findings in fulminant and acute myocarditis. J Am Coll Cardiol 2000;36:227-32.

7 Aoyama N, Izumi T, Hiramori K, and Japanese Investigators of Fulminant Myocarditis, et al. National survey of fulminant myocarditis in Japan: therapeutic guidelines and long-term prognosis of using percutaneous cardiopulmonary support for fulminant myocarditis (special report form a scientific committee). Circ J 2002;66:133-44.

8 Reiss N, el-Banayosy A, Posival H, et al. Management of acute fulminant myocarditis using circulatory support systems. Artif Organs 1996;20:964-70. 